\title{
GESTÃO DA QUALIDADE EM SERVIÇOS POR RESULTADOS E EFICIÊNCIA NA ADMINISTRAÇÃO PÚBLICA: UM ESTUDO DE CASO DE INDICADORES NO INSTITUTO NACIONAL DO SEGURO SOCIAL
}

\section{RESUMO}

O objetivo deste estudo é avaliar a influência da quantidade de servidores e o tempo de serviço sobre indicadores do BSC. Definiu-se como problema de pesquisa: "Qual a relação da quantidade de servidores e o tempo de serviço nos índices de desempenho das agências da Previdência Social?". Os dados utilizados referem-se às 17 agências da Previdência Social vinculadas à Gerência Executiva de Cascavel. Elaboraram-se, para a análise, quatro hipóteses de pesquisa. Utilizaram-se dados do Plano de Ação de cada agência, posteriormente consolidados em planilha eletrônica. Utilizou-se do software SPSS Statistics, para análises estatísticas. Procedeu-se à aplicação do reste Shapiro-Wilk, para análise da normalidade, por se tratar de teste adequado para amostras reduzidas (n < 30). Ato contínuo, efetuou-se o teste de correlação de Spearman. Da análise dos resultados, todas as hipóteses foram refutadas, indicando que a variação na quantidade de servidores ou de tempo de serviço não apresentam mudanças significativas nos índices do BSC.

Palavras-chave: Qualidade em Serviços. Eficiência. Indicadores.

Vinicius Abilio Martins viniciusabilio@gmail.com Doutorando em Contabilidade pela Universidade Federal de Santa Catarina (UFSC). Professor de Ciências Contábeis da Universidade Estadual do Oeste do Paraná (UNIOESTE).

\footnotetext{
Jair Jeremias Junior jair.jeremias.j@gmail.com Mestre em Administração pela Pontificia Universidade Católica do Paraná (PUC/PR). Professor de Administração da Universidade Estadual do Oeste do Paraná (UNIOESTE). Administrador na Universidade Federal da Integração.
}

Serviço Público. Balanced Scorecard.

\section{INTRODUÇÃO}

O serviço público no Brasil apresenta algumas características e peculiaridades que o fazem sistematicamente distinto do setor privado. Está fortemente fundamentado em leis e regras que, segundo Schapiro (2017), são pertencentes a um ramo do Direito chamado de Direito Administrativo, o qual rege, direciona e restringe as ações e reações do serviço público como um todo.

No serviço público, a execução das atividades é delegada a servidores, em sua maioria, integrantes efetivos dos quadros funcionais, à exceção daqueles que adentraram por meio de cargos de livre nomeação e exoneração, chamados de cargos de confiança. Esses serviços públicos, por serem atividades inerentes ao Estado, não admitem concorrência, e o fato de serem desempenhados por 
servidores, conforme legislação, estáveis, por vezes, faz que o serviço prestado não esteja de acordo com o esperado pela população.

Os órgãos pertencentes ao serviço público têm função de servir à sociedade no sentido de garantir seus direitos, prestando serviços no interesse da população em áreas essenciais, devendo, até para cumprir sua missão, prestar serviços de qualidade à população, também, por esta população ser a base de sustentação e a razão de existência do serviço público (MARTINS et al., 2016). A administração pública, sendo a maneira de manter o controle, as metas e o bom andamento dos órgãos públicos em geral, (SOUZA JUNIOR; SILVA, 2016), é gerenciada por gestores profissionais, "eleitos" para desempenhar as atividades necessárias à subsistência e à criação de valor social e/ou ambiental (ENCISO; MARTINS, 2017).

Nas últimas duas décadas, a adoção de novas práticas de gestão no setor público aumentou à medida que as organizações desse setor procuraram melhorar a eficiência, a eficácia e a responsabilidade pública (MARAN; INGLIS; BRACCI, 2017). Criaram-se diversas iniciativas em busca da qualidade, passando por programas que fazem uso de indicadores estabelecidos a partir de nível de qualidade pretendida às partes interessadas (O'BOYLE, 2015), estipulando-se metas para cada um destes a serem alcançadas de suas mais variadas maneiras, envolvendo princípios gerenciais voltados para a eficiência, eficácia e qualidade nos gastos públicos (QUINTAL et al., 2014; PAULA, 2005), guardando cada especificidade relacionada ao serviço.

Um exemplo de ferramenta com indicadores de desempenho como base é o Balanced Scorecard (BSC) de Kaplan e Norton (1997), ao qual se associou o conceito de painel de indicadores com a estratégia da organização, em que se buscou a integração e o balanceamento dos indicadores, visando a um acompanhamento em tempo real dos resultados e oferecendo à organização subsídios para a tomada de decisão referente a melhorias internas, alocações de recursos, entre outros.
O Balanced Scorecard, desenhado por Kaplan e Norton em 1992, e seus derivados são as ferramentas de gerenciamento de desempenho mais utilizadas no ambiente empresarial mais amplo; elas também foram adotadas por outras organizações fora do setor com fins lucrativos (O’BOYLE, 2015).

Esta foi a ferramenta utilizada na Previdência Social, partindo da elaboração de um mapa estratégico que traduz o planejamento estratégico da instituição, o qual engloba missão, visão, valores, perspectivas, direcionadores estratégicos e objetivos estratégicos, representados por diagramas de causa e efeito.

No entanto, apesar de o fator humano ter aumentado sua importância e apresentar-se como um fator chave para aumentar a flexibilidade e o desempenho das organizações (ROCHA; PASSADOR; SHINYASHIKI, 2017), a gestão da Previdência Social, no âmbito do Instituto Nacional de Seguridade Social (INSS), tem pautado suas medidas de desempenho, bem como metas, indicadores ou quaisquer políticas de gestão, predominantemente com base no número de servidores que cada agência da Previdência Social (APS) possui e o tempo de serviço destes servidores.

Apesar de o número de servidores atuantes ser uma preocupação no serviço público, em que a falta de servidores pode ser preocupante (SCOTTON et al., 2013), este não pode ser um dos únicos condicionantes nas avaliações de desempenho. Apesar de, para muitas instituições públicas nacionais, o tempo de serviço ser, muitas vezes, mais valorizado para a progressão na carreira, não se pode generalizar o mérito e a entrega de cada funcionário dentro da organização (ROCHA; PASSADOR; SHINYASHIKI, 2017).

Neste sentido, surge a seguinte questão norteadora desta pesquisa: "Qual a relação da quantidade de servidores e o tempo de serviço nos índices de desempenho das agências da Previdência Social?". Desta forma, para responder a esse questionamento, o presente estudo tem o objetivo de avaliar quais índices sofrem influência pela quantidade de servido- 
res ou pelo tempo de serviço nas agências da Previdência Social.

Este artigo está estruturado da seguinte forma: Na primeira seção, identifica-se o contexto e a problemática da pesquisa. A seção seguinte apresenta a revisão da literatura, em que se aborda o Serviço Público, a Eficiência no Serviço Público, a Qualidade e Qualidade no INSS. Na terceira seção, são apresentados os Métodos e Procedimentos da pesquisa. A quarta e quinta seções apresentam a Análise dos Resultados e, em seguida, a Conclusão da Pesquisa.

\section{REVISÃO DA LITERATURA}

\subsection{SERVIÇO PÚBLICO}

O Direito administrativo consiste na divisão do Direito cujo foco é o estudo da Administração Pública e da atividade de seus integrantes, que são os órgãos, as entidades, os agentes e as atividades públicas. Seu objetivo é o ordenamento dos fins desejados pelo Estado, sendo este o interesse público. Aquilo que estiver relacionado à Administração Pública e aos seus administrados e servidores estará sobre a égide e será estudado pelo Direito Administrativo (DI PIETRO, 2013).

Costa (2008) apresenta a administração pública dividida entre administração direta e administração indireta, em conformidade com a Constituição Federal. A administração direta consiste no presidente, nos ministérios e nos demais órgãos diretamente subordinados ao presidente da República, sendo os Ministérios exemplos destes; já a administração pública indireta é constituída pelos órgãos descentralizados, tais como as fundações, as autarquias, as sociedades de economia mista e as empresas públicas.

Por ser um dos ramos do Direito, o Direito Administrativo é regido por princípios, estabelecidos na Constituição Federal, que sustentam sua base. Os princípios básicos da Administração Pública são regras gerais de observância permanente e obrigatória para o bom gestor público (ARAÚJO; RODRIGUES, 2012). Di Pietro (2013) define os princípios do
Direito Administrativo como o princípio da Legalidade, da Impessoalidade, da Moralidade, da Publicidade e da Eficiência.

No chamado princípio da Legalidade, as instituições da Administração Pública ficam condicionadas a fazer apenas aquilo que a lei permite, diferentemente dos cidadãos comuns, os quais podem fazer o que a lei não proíbe. Em relação ao princípio da Impessoalidade, os atos praticados pelos agentes públicos no exercício de sua função são imputáveis, não ao servidor que os pratica, mas a entidade ou órgão administrativo da Administração Pública ao qual esteja vinculado.

O princípio da Moralidade reza que os atos da administração devem ser morais. Sempre que, em matéria administrativa, se verificar que o comportamento da administração ou do administrado que com ela se relaciona juridicamente, embora em consonância com a lei, ofende a moral, os bons costumes, as regras de boa administração, os princípios de justiça e de equidade, a ideia comum de honestidade, estará havendo ofensa ao princípio da Moralidade Administrativa. O princípio da Publicidade exige a ampla divulgação dos atos praticados pela Administração Pública, ressalvadas as hipóteses de sigilo previstas em lei.

Para este estudo, foca-se no princípio da Eficiência, que se impõe a todo agente público de realizar suas atribuições com presteza, perfeição e rendimento funcional. "É o mais moderno princípio da função administrativa, que já não se contenta em ser desempenhada apenas com legalidade, exigindo resultados positivos para o serviço público e satisfatório atendimento das necessidades da comunidade e de seus membros." (MEIRELLES, 2003, p. 102). O princípio da eficiência complementa o conceito de meritocracia e é aplicado tanto ao corpo da Administração pública quanto a seus agentes.

\subsection{EFICIÊNCIA NOS SERVIÇOS PÚ- BLICOS}

A Eficiência, um dos princípios da Administração Pública (ARAÚJO; RODRIGUES, 
2012), conceitua-se como algo relacionado à aplicação de recursos e insumos no intuito de obter o melhor custo-benefício na relação existente entre objetivos estabelecidos, recursos e insumos utilizados. Para isso, os recursos e insumos necessitam ser aplicados de forma racional, critério presente na base das organizações administrativas e parte integrante do paradigma dominante na teoria organizacional (COELHO, 1979).

O conceito de eficiência está acompanhado dos temas eficácia e efetividade. Motta (1990, p. 230) apresenta eficiência como a realização de normas e a redução de custos. Eficácia relaciona-se ao atingimento de resultados e à qualidade dos serviços e produtos e tem a função de averiguar se os resultados previstos foram realmente obtidos em termos de quantidade e qualidade. A efetividade alude ao efeito da decisão pública, e sua utilidade é verificar se o programa atende adequadamente às demandas, aos apoios e às necessidades da sociedade.

Daft (1999, p. 39) afirma que "eficiência é um conceito mais limitado que diz respeito aos trabalhos internos da organização." Se uma organização puder conseguir um determinado nível de produção com menos recursos que outra, diz-se que ela é mais eficiente. Eficácia organizacional, conforme Daft (1999) é o grau em que a organização realiza seus objetivos. Gomes (2009) define eficiência como o equilíbrio entre os objetivos atingidos e os recursos e insumos utilizados para tal; eficácia como o reconhecimento se os objetivos foram alcançados no nível de qualidade esperado; e a efetividade, a avaliação se os resultados pretendidos, em termos de efeito social ou econômico, foram atingidos, mas "sem" preocupações quanto aos recursos despendidos.

Uma ação pode-se apresentar de forma eficaz ou efetiva, porém, ineficiente, ao se atingir os produtos ou resultados, porém, com gasto excessivo de recursos, mesmo sendo uma situação totalmente possível. Gomes (2009) afirma que, embora seja possível ser eficaz ou efetivo e não ser eficiente, não é possível ser eficiente e não ser eficaz ou efetivo. A rigor, a depender do objetivo, eficiência abrange eficácia ou abrange efetividade. Eficiência, em um amplo conceito, pode ser utilizado como o balanço entre objetivos atingidos, traduzidos em produtos ou em resultados, e recursos utilizados.

\subsection{QUALIDADE}

Inicialmente, ao se estudar o tema qualidade, faz-se necessária a explicitação dos conceitos e abordagens encontradas na literatura. Juran (1992) parte de um olhar relacionado à minimização de defeitos para melhorar a qualidade no sentido de que a qualidade seria a ausência de deficiências. Crosby (1992) entende qualidade como conformidade; em uma visão mais de produção, atrela o tema a necessidades de seguir as especificações.

Já Feigenbaum (1994) relaciona o conceito de qualidade com a correção de problemas e respectivas causas e diversos fatores relacionados com marketing, projetos, engenharia, produção e manutenção, que impactam na satisfação do usuário.

Para Ishikawa (1993), a qualidade está voltada para desenvolver, projetar, produzir e comercializar um produto de qualidade que é mais econômico, mais útil e sempre satisfatório para o consumidor. Entretanto Deming (1997), em uma abordagem voltada ao cliente, entende a qualidade como sendo tudo o que é percebido como melhoria por este.

Em termos gerais, o conceito de qualidade é associado à percepção nas dimensões dos clientes e agentes internos, considerando a conformidade e as especificações e, principalmente, a satisfação do consumidor. Ainda, a preocupação com a qualidade, considerada como um dos valores organizacionais, pode ser encontrada nas maiores instituições brasileiras (JEREMIAS JUNIOR; MARTINS, 2017).

Relacionado a qualidade de serviços, Gronroos (1983) entende que esta pode ser dividida em duas dimensões - qualidade técnica e qualidade funcional, sendo obtida somente por meio da combinação destas duas. Por qualidade técnica, entende-se aquilo que é fornecido pelo funcionário durante o processo de execução do 
serviço, enquanto a qualidade funcional refere-se ao serviço que é fornecido pelo funcionário. A primeira pode compreender o conhecimento tácito do funcionário, know-how, equipamento técnico utilizado e soluções técnicas implementadas. O último tem foco nas contribuições interpessoais do funcionário alinhadas com a prestação do serviço.

Gronroos (1983) parte de uma abordagem de interação do cliente com o serviço e de expectativas normativas associadas a ele, envolvendo o que o funcionário deve fazer e como se deve comportar durante a prestação do serviço, o mesmo ocorrendo com relação à sua posição de cliente. Já Parasuraman, Zeithaml e Berry (1991) entendem a qualidade de um serviço em uma visão de expectativa do cliente, definindo-a como a diferença entre a expectativa do que deve ser fornecido pelo serviço e o desempenho percebido na execução.

Derivada da teoria de Parasuraman, Zeithaml e Berry (1991), originaram-se métricas que visam mensurar a qualidade percebida do serviço. Inúmeros testes de confiabilidade e validade foram efetuados, culminando na elaboração de uma escala de mensuração composta de 22 itens (PARASURAMAN; ZEITHAML; BERRY, 1991), que tem a intenção de medir cinco dimensões da qualidade do serviço:
a) confiabilidade;
b) tangibilidade;
c) empatia;
d) responsividade;
e) garantia.

A escala supracitada é um dos instrumentos mais utilizados para fins de mensuração da percepção do cliente referente à qualidade de serviços, sendo denominada de SERVQUAL, utilizada em diversos casos e com várias adaptações. Nesta e em outras situações, a qualidade do serviço normalmente é associada a percepções, seja do cliente externo ou interno sobre o serviço, sendo esta a referência a ser cruzada com o serviço ou produto final elaborado e servindo, portanto, como base para uma impressão positiva ou negativa sobre o produto, serviço.

A utilização de indicadores de desempe- nho faz parte de uma gestão da qualidade dos serviços no momento em que fornece subsídios à organização para a tomada de decisão no sentido de aprimorar algo que esteja aquém dos resultados esperados, melhoria dos serviços já existentes ao mensurar o que fora efetivamente realizado, confrontando-o com as expectativas relacionadas ao que é entendido como o adequado para a realização do serviço.

Nas últimas duas décadas, a adoção de novas práticas de gestão no setor público aumentou à medida que as organizações do setor público procuraram melhorar a eficiência, eficácia e responsabilidade pública (MARAN; INGLIS; BRACCI, 2017). É um componente integral da capacidade de uma organização oferecer um serviço de qualidade às suas partes interessadas (O’BOYLE, 2015). Os governos estão demonstrando crescente interesse na medição de desempenho no setor público, assim como a imprensa e mídia; e as autoridades estão usando as metas de desempenho e tabelas de classificação, a fim de empurrar, por meio de programas de modernização, e demonstrar que o dinheiro dos contribuintes está sendo entregue (MICHELI; NEELY, 2010). De acordo com Longenecker e Nykodym (1996), as organizações do setor público nos EUA têm sido solicitadas a melhorar sua eficiência, ou seja, prestar mais serviços por dólar e, ao mesmo tempo, demonstrar aumento dos níveis de $a c$ countability para os contribuintes.

Um exemplo de ferramenta que tem indicadores de desempenho como base é o Balanced Scorecard (BSC), de Kaplan e Norton (1997), ao qual se associou o conceito de painel de indicadores com a estratégia da organização, buscando a integração e o balanceamento dos indicadores, visando a um acompanhamento em tempo real dos resultados e oferecendo à organização subsídios para a tomada de decisão referente a melhorias internas, alocações de recursos, entre outros.

Além disso, apesar da inicial proposição ao ambiente empresarial, o BSC mostrou-se capaz de atender às necessidades relacionadas ao setor público, em que a prestação de servi- 
ços de qualidade necessita de inserção e relacionamento com a sociedade, esta financiadora de suas atividades, e à regulamentação legal, por estar vinculado aos Governos instituidores (MARTINS, 2015).

Pesquisas anteriores apresentaram a utilização do BSC no âmbito público a partir de escores balanceados (BOYNE, 2003; MENDES et al., 2012; LIN, 2013; ABDEL-MAKSOUD et al., 2015; MARAN; INGLIS; BRACCI, 2017). No entanto, devido às especificidades apresentadas pelo contexto deste estudo, novas pesquisas como estas se fazem necessárias.

\subsection{1 Índices de Qualidade e Eficiência no âmbito do INSS}

O Instituto Nacional da Seguridade Social (INSS), por sua característica sui generis, não permite a utilização de indicadores similares aos encontrados na literatura, tampouco aqueles utilizados pelas demais instituições públicas ou privadas. A própria organização instituiu seus indicadores, de acordo com suas identificações do que vem a ser qualidade. Nesse sentido, são elencados os índices IRES - Índice de Resolutividade, PRA45 - Porcentagem de processos com análise acima de 45 dias, TMC - Tempo médio de concessão, TMEA Tempo médio de espera do atendimento agendado, TMEA-PM - Tempo médio de espera do atendimento agendado - Perícia Médica, Quantidade de benefícios requeridos, IMA-GDASS - Idade média do acervo, TMD - Tempo médio de decisão, TMI - Tempo médio de indeferimento Quantidade Servidores Ativos, Quantidade de funcionários de carreira administrativa, Quantidade de peritos médicos, Quantidade de servidores com menos de 25 anos de instituição e Quantidade de servidores com mais de 25 anos de instituição.

A Idade média do acervo, IMA, tem o intuito de medir o número de dias entre a data da entrada de um requerimento e a data de seu despacho, favorável ou não. Apresenta seu cálculo pela soma dos tempos de represamento de benefícios dividido pelo total de benefícios re- presados, com periodicidade mensal.

O índice PRA45D representa a porcentagem de processos em análise acima de 45 dias. Este indicador visa medir os processos em análise há mais de 45 dias em relação à demanda mensal da unidade. O desejável é que este índice seja o menor possível.

O Tempo Médio de Espera no Atendimento Agendado - TMEA - visa aferir o tempo que o cidadão aguarda entre a data de solicitação do agendamento e a data de marcação do atendimento, bem como a capacidade da APS em recepcionar toda sua demanda dentro de um limite de tempo aceitável. É apurada a soma dos tempos compreendidos entre a data da solicitação do agendamento até a data do atendimento dividido pela quantidade de agendamentos marcados. Sua periodicidade é apurada mensalmente; quanto menor for sua avaliação, melhor.

O TMEA tem o propósito de aferir o tempo em que o cliente aguarda para ser atendido, bem como a capacidade da APS em recepcionar toda sua demanda dentro de um limite de tempo aceitável. O TMEA-PM visa aferir o tempo que o cidadão aguarda entre a data de solicitação do agendamento da Perícia Médica e a data de marcação do exame, cujo tempo é apurado em dias. Os critérios de ponderação são: $\mathrm{O}$ acompanhamento do histórico do indicador; A pesquisa de satisfação do usuário; A determinação legal de que a conclusão do processo deve ser em até 45 dias.

O Tempo Médio de Concessão - TMC tem o propósito de medir o tempo médio de análise para processos com despacho favorável (concessão). Sua forma de cálculo compreende a soma dos tempos de processamento dos benefícios contados a partir da Data do Deferimento do Benefício menos a Data da Entrada do Requerimento dividido pela Quantidade de benefícios concedidos. Sua periodicidade é mensal, com tendência de quanto menor for este índice, melhor. O propósito é medir o tempo de espera para processos com despacho favorável.

Em similaridade ao tempo médio de concessão, apresentam-se os índices de tempo médio de indeferimento e o tempo médio de decisão. 
O primeiro tem o propósito de medir o tempo médio de análise para processos com despacho desfavorável (indeferimento). $\mathrm{O}$ segundo tem o propósito de medir o tempo médio de análise para processos como um todo, indiferentemente de ser favorável ou desfavorável.

\section{MÉTODOS E PROCEDIMENTOS}

Este trabalho teve como objetivo analisar a influência dos indicadores quantidade de servidores ou tempo de serviço nos índices de desempenho das agências da Previdência Social. O presente estudo tem cunho explicativo, uma vez que tem o intuito de descobrir e descrever as características do fenômeno em questão (RICHARDSON, 1999).

Em relação à abordagem do problema, esta pesquisa classifica-se como quantitativa, pois seus procedimentos foram realizados por levantamento, uma vez que buscou encontrar relações estatísticas entre quantidade de servidores ou tempo de serviço sobre os índices de desempenho das agências da Previdência Social.

A instituição objeto deste estudo é o Instituto Nacional do Seguro Social (INSS). Uma autarquia do Governo Federal do Brasil que recebe as contribuições para a manutenção do Regime Geral da Previdência Social. Esta é a instituição responsável pelo pagamento de aposentadorias, pensão por morte, auxílio-doença, auxílio-acidente e outros benefícios para aqueles que adquirirem o direito segundo o previsto em lei.

Compete ao INSS a operacionalização do reconhecimento dos direitos da clientela do Regime Geral de Previdência Social (RGPS), que atualmente abrange mais de 40 milhões de contribuintes. O Instituto possui em seu quadro administrativo em torno de 40.000 servidores ativos, lotados em todas as regiões do País, que atendem presencialmente a mais de quatro milhões de pessoas todos os meses. O INSS conta com uma rede altamente capilarizada, de cerca de 1.200 unidades de atendimento, as chamadas Agências da Previdência Social (APS), presentes em todos os estados da Federação.

Os dados utilizados nesta pesquisa refe- rem-se às 17 Agências da Previdência Social (APS) vinculadas à Gerência Executiva (GEX) de Cascavel PR. Essas agências são Assis Chateaubriand, Cascavel, Foz do Iguaçu, Francisco Beltrão, Medianeira, Pato Branco, Realeza, Toledo, Guaíra, Mangueirinha, Marechal Cândido Rondon, Palmas, Santo Antônio do Sudoeste, Dois Vizinhos, Palotina, Coronel Vivida e São Miguel do Iguaçu.

Inicialmente, buscou-se a autorização para a utilização dos dados, sob consulta ao Setor de Atendimento da Gerência Executiva em Cascavel. Posteriormente, tal pedido foi repassado para a Superintendência Regional Sul, localizada na cidade de Florianópolis, Santa Catarina, responsável pelos estados do Paraná, Santa Catarina e Rio Grande do Sul, a qual, por meio da figura de seu superintendente, autorizou a utilização dos dados relativos à Gerência Executiva de Cascavel.

Após autorizado, os dados foram coletados no sistema Plano 2013 (www-plano 2013). Este sistema apresenta os indicadores do Plano de Ação, vinculado ao planejamento estratégico do Instituto. O Plano de Ação estabeleceu prioridades e metas, traduzidas em um conjunto de ações e indicadores alinhados aos objetivos estratégicos do Mapa, visando à concretização das estratégias e o alcance dos resultados definidos para o período. O Sistema de Acompanhamento do Plano de Ação é o módulo de acompanhamento do Plano que, a partir da visualização do Mapa e da seleção de cada um dos objetivos estratégicos, disponibiliza informações e resultados mensais das unidades do INSS, por ação.

Para a análise, elencando os indicadores relacionados à qualidade, aliado às políticas de gestão no âmbito da Previdência Social no Brasil, sob a argumentação da qualidade técnica e funcional propostas por Gronroos, foram propostas 04 hipóteses de pesquisa, a serem testadas: 
HIPÓTESE 01: Agências com mais servidores e com servidores mais experientes apresentariam menores índices de processos com prazo inferior a 45 dias e menor Idade média do acervo - IMA.

HIPÓTESE 02: Servidores com mais tempo de casa apresentariam um índice de resolutividade maior do que aqueles com menos, seja pela experiência ou know-how adquirido ao longo do tempo.

HIPÓTESE 03: O tempo médio de espera no atendimento agendado (TMEA) e o tempo médio de espera do atendimento agendado - Perícia médica (TMEA-PM) são influenciados pela quantidade de servidores, pelo tempo de serviço dos servidores e pela quantidade de benefícios requeridos.

HIPÓTESE 04: Os tempos médios de decisão, concessão e indeferimento são influenciados pela quantidade de servidores ou pela experiência (tempo de serviço) dos servidores.

Quadro 1 - Hipóteses da Pesquisa

Fonte: elaborado pelos autores (2017).

Os dados constantes no Plano de Ação apresentam-se, no sistema corporativo do Instituto, por agência. Posteriormente, foram consolidados os dados de todas as agências objeto do estudo em uma única planilha eletrônica.

Em seguida, com a utilização do software SPSS Statistics, efetuaram-se as análises estatísticas. A primeira análise estatística realizada foi um teste para avaliar a normalidade das variáveis, ou seja, analisar a forma de distribuição dos dados e a adequação para a continuidade das análises estatísticas; visto que, se a variação em relação à distribuição normal for suficientemente grande, todos os testes estatísticos são inválidos conforme Hair Junior et al. (2005). Para que a normalidade das variáveis pudesse ser testada, procedeu-se à aplicação do teste Shapiro-Wilk. O teste Shapiro-Wilk é utilizado para avaliar se as observações possuíam distribuição de retorno próxima da distribuição normal (MAMEDE et al., 2017; SHAPIRO; WILK, 1965), por ser considerado um teste de ajustamento mais robusto para amostras reduzidas $(\mathrm{n}<30)$. Em seguida, para verificar a influência entre variáveis, neste caso nos indicadores do INSS, efetuou-se o teste de correlação de Spearman, sendo este, um teste de correlação não paramétrico.

\section{ANÁLISE DOS DADOS}

Para a análise dos dados, foram utilizados os indicadores de qualidade que o INSS utiliza em seu mapa estratégico. Os indicadores analisados foram os seguintes: IRES - Índice de Re- solutividade, PRA45 - Porcentagem de processos com análise acima de 45 dias, TMC - Tempo médio de concessão, TMEA - Tempo médio de espera do atendimento agendado, TMEA-PM Tempo médio de espera do atendimento agendado - Perícia Médica, Quantidade de benefícios requeridos, IMA-GDASS - Idade média do acervo, TMD - Tempo médio de decisão, TMI - Tempo médio de indeferimento, Quantidade de Servidores Ativos, Quantidade de funcionários de carreira administrativa, Quantidade de peritos médicos, Quantidade de servidores com menos de 25 anos de instituição e Quantidade de servidores com mais de 25 anos de instituição.

HIPÓTESE 01: Agências com mais servidores e com servidores mais experientes apresentariam menores indices de processos com prazo inferior a 45 dias e menor Idade média do acervo - IMA.

HIPÓTESE 02: Servidores com mais tempo de casa apresentariam um índice de resolutividade maior do que aqueles com menos, seja pela experiência ou know-how adquirido ao longo do tempo.

$\mathrm{Na}$ análise de correlações, o único índice que não apresentou sensibilidade aos demais foi o de Resolutividade. Este índice apresenta o percentual de processos que obtiveram sua resolução e conclusão no dia em que foram protocolados. A meta estipulada para este índice é de atingimento de 45 por cento dos processos concluídos na mesma data do protocolo para o primeiro semestre de 2013 e 50 por cento dos processos concluídos na data do protocolo para o segundo semestre. 
Considerando que os dados são referentes a Julho/2013, aplica-se o percentual de 50 por cento. Das 17 agências objeto do estudo, 08 delas apresentaram índice superior à média desejada e 09 inferior, com uma média de 46 por cento, em um intervalo de $22,31 \%$ e $58,64 \%$. Em relação ao tempo de serviço, aproximadamente $85,6 \%$ dos servidores têm até 25 anos de serviço e 14,4\% têm mais de 25 anos. O fato de não apresentar correlação com os demais índices, denota que a eficiência, neste indicador, não é afetada pelas variáveis independentes relacionados aos servidores, refutando, assim, a hipótese (número de servidores e tempo de instituição).

Os índices IMA e PRA45D não apresentaram sensibilidade quanto ao número de servidores, nem em relação ao tempo de serviço deles. Ambos os índices apresentaram as mesmas correlações, sendo estas Tempo médio de concessão, Tempo médio de indeferimento, Tempo médio de decisão e Tempo médio de espera do atendimento agendado.

HIPÓTESE 03: O tempo médio de espera no atendimento agendado (TMEA) e o tempo médio de espera do atendimento agendado - Perícia médica (TMEA-PM), são influenciados pela quantidade de servidores, pelo tempo de serviço dos servidores e pela quantidade de beneficios requeridos.

Nas correlações, este índice apresentou significância com os índices Porcentagem de processos com análise acima de 45 dias, Tempo médio de concessão, Tempo médio de espera do atendimento agendado - Perícia Médica, Tempo médio de tramitação de processos de início de recursos administrativos, Tempo médio de agendamento ativo, Tempo médio de decisão e Tempo médio de indeferimento, apresentando correlação mais forte com o índice de Idade média do acervo. Assim, o TMEA não apresentou correlação com a quantidade de servidores ou com o tempo de serviço dos servidores, nem pela quantidade de benefícios requeridos.

HIPÓTESE 04: Os tempos médios de decisão, concessão e indeferimento são influenciados pela quantidade de servidores ou pela experiência (tempo de serviço) dos servidores.
Para os três índices, houve correlações significativas com os seguintes índices: Porcentagem de processos com análise acima de 45 dias, Tempo médio de espera do atendimento agendado, Tempo médio de espera do atendimento agendado - Perícia Médica, Idade média do acervo e Tempo médio de agendamento ativo - Perícia médica.

Ao apresentar as mesmas correlações, infere-se que esses índices apresentam as mesmas influências, referentes aos índices de tempo médio de espera e influenciam os mesmos índices, referentes aos prazos de 45 dias e idades do acervo. No tocante à quantidade de servidores ou ao tempo de serviço dos servidores, foi observado que não houve correlação entre estes e os índices acima.

No que tange à quantidade de benefícios requeridos, este índice apresentou relação com os seguintes, Quantidade de servidores ativos, Quantidade de funcionários de carreira administrativa, Quantidade de peritos médicos, Quantidade de servidores com menos de 25 anos de instituição e Quantidade de servidores com mais de 25 anos de instituição.

Em Quantidade de servidores ativos, suas relações foram com os índices de Quantidade de benefícios requeridos, Quantidade de funcionários de carreira administrativa, Quantidade de peritos médicos, Quantidade de servidores com menos de 25 anos de instituição e Quantidade de servidores com mais de 25 anos de instituição.

\section{CONCLUSÃO}

O objetivo deste artigo foi avaliar quais índices de desempenho da Previdência Social sofrem influência pela quantidade de servidores ou tempo de serviço nas agências da Previdência Social e quais índices independem dessas variáveis. Para se poder alcançar este objetivo, apresentou-se como problema de pesquisa: "Qual a relação da quantidade de servidores e tempo de serviço nos índices de desempenho das agências da Previdência Social?."

No intuito da resolução do problema de 
pesquisa apresentado, foram abordadas quatro hipóteses de pesquisa, relacionando números de servidores por agência e tempo de serviço / experiência dos servidores com os indicadores utilizados, por meio do BSC, pela Previdência Social, tais quais os índices de processos com prazo inferior a 45 dias e menor média de Idade do acervo - IMA, índice de resolutividade, tempo médio de espera no atendimento agendado (TMEA) e o tempo médio de espera do atendimento agendado - perícia médica (TMEA-PM) e tempos médios de decisão, concessão e indeferimento.

Por meio das análises realizadas, concluiu-se que nenhum dos índices apresentados nas hipóteses sofrem influência pela quantidade de servidores ou tempo de serviço nas agências da Previdência Social. Dessa forma, todas as hipóteses foram refutadas. A quantidade de benefícios requeridos apresentou relação com os indicadores de Quantidade de servidores ativos, Quantidade de funcionários de carreira administrativa, Quantidade de peritos médicos, Quantidade de servidores com menos de 25 anos de instituição e Quantidade de servidores com mais de 25 anos de instituição. A Quantidade de servidores ativos apresentou relações com os índices de Quantidade de benefícios requeridos, Quantidade de funcionários de carreira administrativa, Quantidade de peritos médicos, Quantidade de servidores com menos de 25 anos de instituição e Quantidade de servidores com mais de 25 anos de instituição. Ambos os indicadores se correlacionam, demonstrando que, na tomada de decisão da lotação das Agências da Previdência Social, é levada em consideração a quantidade de benefícios solicitados.

Em relação ao Índice de resolutividade, este não apresentou relação alguma com os demais índices estudados. O fato de não apresentar correlação com os demais índices, denota que a eficiência, neste indicador, não é afetada pelas variáveis independentes relacionadas ao número de servidores e tempo de instituição. Para uma análise mais aprofundada deste indicador, sugere-se que seja utilizada uma pesquisa qualitativa, com a utilização de entrevistas com os servidores responsáveis pelo desempenho deste índice.

Não houve diferenças significativas entre os índices correlacionados de Quantidade de servidores com menos ou mais de 25 anos de instituição. $\mathrm{O}$ fato de possuir maior tempo na instituição não necessariamente influencia os índices de forma diferente daqueles com menos tempo de instituição. Por fim, vale ressaltar que existe uma correlação entre a quantidade de benefícios requeridos e o número de servidores por agência. Conclui-se, assim que, de certo modo, a quantidade de servidores está equalizada com a demanda existente de trabalho nas unidades.

Quanto às limitações desta pesquisa, ressalta-se a utilização de um conjunto de Agências da Previdência Social (APS), não sento utilizado o total de agências localizadas no Brasil, devido às dificuldades operacionais com os dados. Além desta, indicadores considerados sigilosos não puderam ser utilizados, devido à não autorização por parte da Instituição, o que limita o poder explicativo desta pesquisa.

Como sugestão de pesquisas futuras, recomenda-se uma pesquisa longitudinal, em que os indicadores utilizados nesta pesquisa podem sofrer influência no decorrer dos períodos; pesquisas relacionadas a outros órgãos da Administração Pública Brasileira, como a Receita Federal do Brasil, instituições universitárias e instituições hospitalares, sendo estas as de maior contato com a população, cuja gestão de qualidade do serviço pode apresentar o melhor retorno à sociedade.

\section{QUALITY MANAGEMENT IN SERVICES BY RESULTS AND EFFICIENCY IN PUBLIC ADMINISTRATION: A CASE STUDY OF INDICATORS IN THE NATIONAL INSTITUTE OF SOCIAL SECURITY}

\section{ABSTRACT}

The objective of this study is to evaluate the influence of the number of public servants and 
time of employment on BSC indicators. The following question was defined as the main research problem: "What is the relation of the number of public servants and time of employment in the performance indexes of Social Security agencies?". The data used refers to the 17 Social Security Agencies linked to the Executive Management of Cascavel. For the analysis, four research hypotheses were put forth. We used data from the Action Plan of each agency, later consolidating it in electronic spreadsheets. SPSS Statistics software was used for statistical analysis. The Shapiro-Wilk substrate was applied for the analysis of normality, since it is an adequate test for reduced samples (n $<30$ ). Then, the Spearman correlation test was performed. From the analysis of the results all the hypotheses were refuted, indicating that the variation in the number of public servants or time of employment did not present significant changes in the BSC indexes.

Keywords: Quality in Services. Efficiency. Indicators. Public servisse. Balanced Scorecard.

\section{GESTIÓN DE LA CALIDAD EN SERVICIOS POR RESULTADOS Y EFICIENCIA EN LA ADMINISTRACIÓN PÚBLICA: UN ESTUDIO DE CASO DE INDICADORES EN EL INSTITUTO NACIONAL DE SEGURO SOCIAL}

\section{RESUMEN}

El objetivo de este estudio es de evaluar la influencia de la cantidad de servidores y del tiempo de servicio sobre indicadores del BSC. Se definió como problema de investigación: "Cual la relación de la cantidad de servidores y tiempo de servicio en los índices de desarrollo de las agencias de la Previdencia Social?". Los datos utilizados se refieren a las 17 Agencias de la Previdencia Social vinculadas a la Gerencia Ejecutiva de Cascavel. Se elaboró, para el análisis, cuatro hipótesis de investigación. Se utilizaron datos del Plan de Acción de cada agencia, posteriormente consolidados en una hoja de cálculo electrónica. Se utilizó el software SPSS Statistics, para el análisis estadístico. Se procedió a la aplicación del substrato Shapiro-Wilk, para el análisis de la normalidad, por tratarse de test adecuado para muestreos reducidos $(\mathrm{n}<30)$. A seguir, se efectuó el test de correlación de Spearman. Por el análisis de los resultados todas las hipótesis fueron refutadas, indicando que la variación en la cantidad de servidores o de tiempo de servicio no presenta cambios significativos en los índices del BSC.

Palabras-clave: Calidad en servicios. Eficiencia. Indicadores. Servicio público. Balanced Scorecard.

GESTION DE LA QUALITE EM SERVICES PAR RESULTATS ET EFFICACITE DANS

L'ADMINISTRAITON PUBLIQUE : UNE ETUDE DE CAS DES INDICATEURS DANS L'INSTITUT NATIONAL DE LA SECURITE SOCIAL

\section{RESUME}

L'objectif de cette étude est d'évaluer l'influence du nombre de fonctionnaires et de leur temps de service sur les indicateurs du BSC. Le problème de recherche fût défini comme : "Quelle est la relation entre le nombre de fonctionnaires et leurs temps de service sur les indicateurs de développement des agences de la Sécurité Sociale ? ». les données utilisées se réfèrent aux 17 agences de la Sécurité Sociale attachées à la Direction Exécutive de Cascavel. Quatre hypothèses d'investigation ont été formulées. Nous avons utilisés les données du Plan d'Action de chaque agence, que nous avons $a$ posteriori consolidées sur des feuilles de calcul eléctroniques. Nous avons utilisé le logiciel SPSS Statistics pour l'analyse statistique. Nous vanos appliqué le test Shapiro-Wilk, pour anali- 
ser la normalité, puisque c'est un test approprié pour un échantillonage reduit $(\mathrm{n}<30)$. En suite, nous avons effectué le test de corrélation de Spearman. Après l'analyse des résultats, toutes les hypotèses ont été rejettées, indicant que la variation du nombre de fonctionnaire ou du temps de service ne présentent pas des changements significatifs sur les indicateurs du BSC.

Mots-clés: Qualité en services. Efficacité. Indicateurs. Service public. Balanced Scorecard.

\section{REFERÊNCIAS}

ABDEL-MAKSOUD, Ahmed et al. The use of performance information in strategic decision making in public organizations. International Journal of Public Sector Management, United Kingdom, v. 28, n. 7, p. 528-549, 2015.

ARAÚJO, L. M.; RODRIGUES, M. I. A. A relação entre os princípios da eficiência e da economicidade nos contratos administrativos. Revista do Serviço Público, Brasília, v. 63, n. 1, p. 43-62, 2012.

BOYNE, George A. What is public service improvement?. Public Administration, United Kingdom, v. 81, n. 2, p. 211-227, 2003.

COELHO, Edmundo Campos. A retórica da racionalidade e o mito da estrutura. [S.1.]: IUPERJ, 1979.

COSTA, Frederico Lustosa da. Brasil: 200 anos de Estado; 200 anos de administração pública; 200 anos de reformas. Revista de Administração Pública, Rio de Janeiro, v. 42, n. 5, p. 829874, 2008.

CROSBY, PB. Quality is free: the art of making quality certain. [S.1.]: Mentor Books, 1992.

DAFT, Richard L. Teoria e projetos das organizações. 6. ed. Rio de janeiro: LTC, 1999.

DEMING, W. E. A nova economia para a in- dústria, o governo e a educação. Tradução Heloisa Martins Costa. Rio de Janeiro: Qualitymark, 1997.

DI PIETRO, Maria Sylvia Zanella. Direito administrativo. 26. ed. São Paulo: Atlas, 2013.

ENCISO, Luis Fernando; MARTINS, Vinicius Abilio. Estrutura, tecnologia e o efeito da configuração estrutural no desempenho das organizações: um ensaio teórico. Revista Metropolitana de Governança Corporativa, São Paulo, v. 2 , n. 1, p. $38-53,2017$.

FEIGENBAUM, A. V. Controle da qualidade total: gestão e sistemas. Tradução Regina Cláudia Loverri. São Paulo: Makron Books, 1994. $1 \mathrm{v}$

GOMES, Eduardo Granha Magalhães. Gestão por Resultados e eficiência na Administração Pública: uma análise à luz da experiência de Minas Gerais. 2009. 187 f. Tese (Doutorado em Administração Pública e Governo) - Escola de Administração de Empresas de São Paulo FGV, São Paulo, 2009.

GRONROOS, Christian. Strategic management and marketing in the service sector. Cambridge, MA: Marketing Science Institute, 1983.

HAIR JUNIOR, J. F. et al. Análise multivariada de dados. 5. ed. Porto Alegre: Bookman, 2005.

ISHIKAWA, K. Controle de qualidade total: à maneira japonesa. 2. ed. Rio de Janeiro: Campus, 1993.

JEREMIAS JUNIOR, Jair; MARTINS, Vinicius Abilio. Perfis de valores organizacionais: uma análise das 50 maiores empresas privadas brasileiras. Caderno Profissional de Administração da UNIMEP, São Paulo, v. 7, n. 1, p. 101-117, 2017. 
JURAN, J. M. A qualidade desde o projeto: os novos passos para o planejamento da qualidade em produtos e serviços. São Paulo: Pioneira, 1992.

KAPLAN, Robert S.; NORTON, David P. A estratégia em ação: balanced scorecard. Rio de Janeiro: Campus, 1997.

LIN, Wen-Cheng. Balanced scorecard and IPA enables public service in township management: local government performance. Lex Localis, Slovenia, v. 11, n. 1, p. 21, 2013.

LONGENECKER, Clinton O.; NYKODYM, Nick. Public sector performance appraisal effectiveness: a case study. Public Personnel Management, United States, v. 25, n. 2, p. 151-164, 1996.

MAMEDE, S. P. N. et al. Empresas brasileiras estatais e não estatais: uma análise das relações de endividamento. Revista Evidenciação Contábil \& Finanças, Paraíba, v. 5, n. 2, p. 4-22, 2017.

MARAN, Laura; INGLIS, Robert; BRACCI, Enrico. Examining the process of performance measurement system design and implementation in two Italian public service organizations. Financial Accountability \& Management, United States, v. 33, n. 4, p. 406-421, 2017.

MARTINS, Vinicius Abilio. Proposta de um mapa estratégico para uma universidade pública. Revista Evidenciação Contábil \& Finanças, Paraíba, v. 3, n. 2, p. 88-103, 2015.

MARTINS, V. A. et al. Teoria de agência aplicada no setor público. Espacios, Caracas, v. 37 , n. 35, p. $24,2016$.

MEIRELLES, Hely Lopes. Direito administrativo brasileiro. São Paulo: Malheiros, 2003.

MENDES, Paula et al. The balanced scorecard as an integrated model applied to the Portuguese public service: a case study in the waste sector. Journal of Cleaner Production, v. 24, p. 20-29, 2012.

MICHELI, Pietro; NEELY, Andy. Performance measurement in the public sector in England: searching for the golden thread. Public Administration Review, United Kingdom, v. 70, n. 4, p. 591-600, 2010.

MOTTA, Paulo Roberto. Avaliação da administração pública: eficiência, eficácia e efetividade. [S.1.]: FUNDAP, 1990.

O'BOYLE, Ian. Developing a performance management framework for a national sport organisation. Sport Management Review, Australia, v. 18, n. 2, p. 308-316, 2015.

PARASURAMAN, A.; ZEITHAML, Valarie; BERRY, Leonard. Refinement and reassessment of the SERVQUAL scale. Journal of Retailing, United Kingdom, v. 67, n. 4, p. 420450, 1991.

PAULA, Ana Paula Paes de. Administração pública brasileira entre o gerencialismo e a gestão social. RAE-revista de administração de empresas, São Paulo, v. 45, n. 1, p. 36-49, 2005.

QUINTAL, Renato Santiago et al. Novos formatos organizacionais na administração pública: o caso das organizações militares prestadoras de serviço. Revista Gestão em Análise, Fortaleza, v. 3, n. 1/2, p. 47-59, 2014.

RICHARDSON, R. J. Pesquisa social: métodos e técnicas. 3. ed. São Paulo: Atlas, 1999.

ROCHA, G. B.; PASSADOR, C. S.; SHINYASHIKI, G. T. What is the social gain from competency management? the employees' perception at a brazilian public university. Revista de Administração, São Paulo, v. 52, n. 3, p. 233-245, 2017. 
SCHAPIRO, M. G. Legalidade ou discricionariedade na governança de bancos públicos: uma análise aplicada ao caso do BNDES. Revista de Administração Pública, Rio de Janeiro, v. 51, n. 1, p. 105-128, 2017.

SCOTTON, R. F. et al. Gestão de projetos de turismo envolvendo recursos federais: o caso de Bento Gonçalves-RS. Revista Ciências Administrativas, Fortaleza, v. 19, n. 2, p. 741775, 2013.

SHAPIRO, S. S.; WILK, M. B. An analysis of the variance test for normality. Biometrika, United Kingdom, v. 52, n. 3, p. 591-611, 1965.

SOUZA JUNIOR, M.; SILVA, M. Z. Gestão Pública Estadual: percepção dos gestores sobre a qualidade dos controles internos. Revista Catarinense da Ciência Contábil, Florianópolis, v. 15, n. 46, p. 47-60, 2016. 